
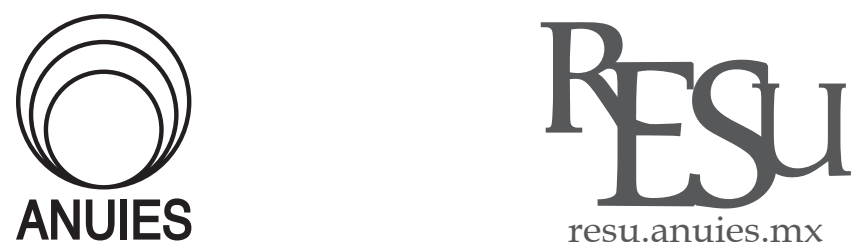

REVITA AE ${ }^{2}$
EDICACIÓN
SUPERIOR

ARTíCULO

\title{
Investigación narrativa para contar historias: líneas de vida de estudiantes universitarios con discapacidad
}

\author{
Narrative research to tell stories: life lines of university students with \\ disabilities
}

\author{
Almudena Cotán Fernández* \\ *Universidad de Sevilla, España \\ Correo electrónico: almudenacotanfernandez@gmail.com
}

Recibido el 24 de agosto del 2018; aceptado el 26 de octubre del 2019

\section{Resumen}

En este artículo se presentan los resultados parciales de una investigación realizada en una universidad ubicada en el sur de España cuyo propósito principal es analizar los facilitadores y obstaculizadores que los estudiantes con discapacidad identificaban durante su trayectoria universitaria. A partir del método biográfico, se construyen dos historias de vida utilizando diversas técnicas para recoger datos mediante un análisis narrativo, centrándonos en historias de vidas individuales y concretas. Finalmente, se abordarán las conclusiones obtenidas en este trabajo a través de una discusión centrada en las principales barreras y ayudas que estos alumnos han percibido en su experiencia universitaria.

Palabras clave: Estudiantes con discapacidad; Instituciones de educación superior; Línea de vida; Educación inclusiva; Método biográfico-narrativo 


\begin{abstract}
This article presents a research partial result accomplished in a university located in southern Spain, whose main purpose is to analyze the facilitators and obstacles that students with disabilities identified during their university career. Beginning with a biographical method, two real stories are constructed using different data collection techniques. This narrative analysis will be focus on single stories and concrete lives. Finally, the conclusions obtained in this work will be addressed through a discussion about the main barriers and aids that these students have perceived by their university experience.
\end{abstract}

Keywords: Students with disabilities; Higher education institutions; Life lines; Inclusive education; Biographicalnarrative method.

\title{
Introducción
}

$\mathrm{E}$ acceso de estudiantes con discapacidad en las Instituciones de Educación Superiores (ES) un derecho reconocido legalmente (Moriña \& Cotán, 2017; Yssel, Pak \& Beilke, 2016) y una realidad que se va incrementando en los sucesivos cursos en los diversos contextos nacionales e internacionales. En el caso de España, lugar en el que tiene origen esta investigación, según la Fundación Universia (2017), en el curso 2016/2017 hubo un total de 20.793 estudiantes con alguna discapacidad, lo que supone un $1,4 \%$ del total de estudiantes matriculados. Este aspecto resulta llamativo si se compara con datos de años anteriores, como en el curso 2011/2012 en el que hubo un total de 18.418 (Fundación Universia, 2012) estudiantes con discapacidad matriculados en la Universidad o el curso 2012/2013 en el que la Fundación Universia (2013) constató un total de 19.953 estudiantes. Sin embargo, si nos ocupamos de cursos más cercanos en el tiempo, se puede comprobar cómo en el 2013/2014 hubo un total de 21.913, en el 2014/2015, 21.577, y en el 2015/2016, 20.695 estudiantes con discapacidad matriculados en universidades nacionales (Fundación Universia, 2014, 2015, 2016), datos más similares a los actuales, lo que supone un 1,5\% aproximado de la población universitaria española.

Es preciso reconocer que el derecho de acceso de las personas con discapacidad a la Enseñanza Superior no es algo nuevo, sino que es posible remontarse a 1948, con la Declaración Universal de los Derechos Humanos, en su artículo 26.1, donde se garantiza que las personas con discapacidad pue- 
dan acceder a la Es sin ningún tipo de discriminación. Entre algunas de las Convenciones que han seguido a la Declaración Universal de los Derechos Humanos, se puede citar la Declaración Mundial sobre educación para todos (1990), la Conferencia Mundial sobre Educación Superior (1998), la conferencia Mundial sobre Necesidades Educativas Especiales (1994), así como las Actas de Discriminación de países como Australia (1992), Estados Unidos (1990) o Reino Unido (1995, 2010), y, sobre todo, la Convención sobre los Derechos de las Personas con Discapacidad (2008) donde se recoge de manera clara y explícita en su artículo 24 que los Estados Partes han de asegurar el acceso general de las personas con discapacidad a la educación superior.

Numerosas iniciativas han influido en el acceso de este colectivo de estudiantes en la Universidad, especialmente a nivel legislativo. Si se hace una revisión sobre la política y la normativa actual existentes en educación de las personas con discapacidad, el derecho a la no discriminación y a la igualdad de oportunidades destacan de forma notoria (Ruiz Hidalgo, 2015). Entre la normativa vigente, se puede destacar la europea cuya influencia se ha dejado notar de forma directa en la normativa española (Rodríguez \& Álvarez, 2014) y la propia normativa española que ha velado por garantizar estos derechos de educación al colectivo de personas con discapacidad, como es el caso del Real Decreto Legislativo 1/2013 (Boletín Oficial del Estado [воE], 2013) de derechos de las personas con diversidad funcional y su inclusión social o la Ley Orgánica 4/2007 (воE, 2007) de Universidades en las que se pone de manifiesto la obligación de responder a las necesidades y demandas de este colectivo de estudiantes asegurando, en todo momento, aspectos que garanticen su acceso, su permanencia, su participación y su integración plena en todos los espacios universitarios (Moriña \& Cotán, 2017; Rodríguez \& Álvarez, 2014; Vander Kloet, 2015).

Sin embargo, aunque existe de forma tácita normativa nacional e internacional que vele y garantice los derechos de los estudiantes con discapacidad en las instituciones de ES, en algunos organismos sigue habiendo un vacío legal no promoviendo en las universidades procesos inclusivos. Por ello, es necesario que además de la normativa legal, las universidades promuevan otro tipo de acciones que no sólo expliquen la presencia del estudiante en la universidad sino que garanticen también su permanencia en un contexto inclusivo. Así, la creación de oficinas, la figura de un tutor, la formación especializada a los docentes en materia de atención a la diversidad o la implementación de prácticas educativas inclusivas (Cruz y Casillas, 2017; Melero, Moriña \& López-Gavira, 2018; Morgado, López y Moriña, 2017), serían aspectos esenciales para alcanzar tal fin. 
En este sentido, actualmente, en España, las políticas educativas y de inclusión que se están llevando a cabo inciden de forma satisfactoria y positiva en la formación de los estudiantes con discapacidad en las universidades. Sin embargo, siguen existiendo problemas de acceso a la educación y de permanencia que limita su proceso de aprendizaje (Cruz y Casillas, 2017; Gibson, 2012; Thomas, 2016; Vickerman \& Blundell, 2010). Numerosos estudios evidencian esta realidad siendo buena muestra de ello los trabajos realizados por Cruz y Casillas (2017), Fossey, Chaffey, Venville, Ennals, Douglas, \& Bigby (2017), Kilpatrick, Johns, Barnes, Fischer, McLennan, \& Magnussen 2017), Ruiz Hidalgo (2015), Strnadová, Hájková, \& Květonová (2015) y Vickerman y Blundell (2010).

Entre los resultados reflejados en estos trabajos, se puede observar cómo los estudiantes con discapacidad tienen que seguir enfrentándose a numerosas y continuas barreras en el entorno institucional, como son la accesibilidad arquitectónica y la inaccesibilidad de los entornos virtuales, procesos burocráticos y administrativos lentos y costosos, así como normativa no aplicada. Por otro lado, se pueden destacar las barreras localizadas en el contexto micro-institucional, como es el caso del aula. Buen ejemplo de ello se puede encontrar en los curricula no inclusivos, docentes inaccesibles o ausencia de modificaciones curriculares, aspectos que tanto por separados como en conjunto dificultan la experiencia universitaria de estos estudiantes, llevando, en algunas ocasiones, al abandono de los estudios universitarios.

Por otro lado, también se deben reseñar experiencias positivas que tienen una gran incidencia e importancia sobre la experiencia y la trayectoria de los estudiantes, como son los compañeros, profesores, concienciación y empatía de la comunidad universitaria, servicios y unidades de apoyo y orientación, tiempo extra en los exámenes, así como el uso de las nuevas tecnologías (Jacklin, Robinson, O’Meara, \& Harris, 2007).

De esta forma se observa cómo la permanencia de este colectivo de estudiantes en las instituciones de ES supone una oportunidad y un empoderamiento de sus propias vidas (Melero, Moriña \& López-Gavira, 2018; Moriña, 2017a; Moswela \& Mukhopadhyay, 2011; Shaw, 2009). Esta experiencia les supone opciones de mejora y de aumento de autonomía en su vida personal pudiendo facilitarles herramientas y estrategias que les permitan, en un futuro, obtener y mantener un trabajo, así como una vida independiente (Guzmán, 2017; Melero, Moriña \& López-Gavira, 2018).

Estos obstáculos nos llevan a pensar, apoyándonos en el modelo social de la discapacidad, que es el contexto el que genera las barreras a las personas con discapacidad y que éstas no son consecuencias de sus barreras personales o individuales, tal y como señalan las premisas del modelo médico 
de discapacidad. En este sentido, tal y como apuntan Baglieri, Valle, Connor y Gallagher (2011) y Jacklin et al., (2007), las dificultades con los que los estudiantes con discapacidad se encuentran en la universidad están en su entorno.

Esta realidad confirma la necesidad de adaptación y reajuste que se hace evidente en los centros de enseñanza superior para que sean totalmente accesibles para toda la comunidad universitaria. Por ello, el objetivo que deben de seguir las políticas y los planes de mejora ha de ser alcanzar la inclusión plena, tomando como referencia el diseño universal. Por ello, se considera importante que las universidades incluyan en sus agendas formativas programas específicos dirigidos a la atención del alumnado con discapacidad para el profesorado que trabaje con estudiantes con discapacidad, ya que, tal y como indica Hurst (2015), la formación en materia de discapacidad debería ser obligatoria. Asimismo, se considera que la meta a seguir debe ser, por tanto, alcanzar la plena inclusión de todos y todas, tomando como referente el diseño universal. En este sentido, estamos de acuerdo con Pliner y Johnson (2004) o Shaw (2009), cuando afirmamos que estas mejoras no sólo van a beneficiar a los estudiantes con discapacidad, sino también al resto del alumnado en general.

Se considera de suma importancia conocer cuáles son las principales barreras y ayudas que los estudiantes con discapacidad identifican en las instituciones de Educación Superior. Por ello, el objetivo del presente artículo es presentar las narraciones de dos estudiantes con discapacidad en la Universidad a través de la técnica de recogida de datos de línea de vida. A través de esta técnica, se detallarán, desde un punto de visto gráfico y narrativo, los hechos más destacados (tanto positivos como negativos) de su trayectoria universitaria.

\section{Línea de vida como herramienta de narración}

Las líneas de vida o las también denominadas graphic life map, life-line o timeline (Brott, 2001; Melero, Moriña, \& López-Gavira, 2018; Okocha, 1998), como técnica de recogida de datos en historias de vida, son representaciones visuales que recogen los acontecimientos, sucesos, situaciones, experiencias o sentimientos de una persona en orden cronológico, pudiendo incluir, de forma posterior o simultánea, interpretaciones de los acontecimientos descritos (Gramling y Carr, 2004). De esta forma, esta técnica permite conocer el pasado y el presente en la historia de los protagonistas, destacando hitos esenciales en la misma que son establecidos por orden cronológico (Gramling, \& Carr, 2004). 
No se puede afirmar que existe un único modelo de línea de vida, ya que podemos encontrar desde aquellas que son lineales y que no aportan ningún dato destacado ni valorable sobre el hecho reseñado, hasta aquellas que incluyen grabaciones audiovisuales si se estiman oportunas (necesidad de ampliar la información o dejarla registrada) (Melero, Moriña, \& López-Gavira, 2018). Sin embargo, hay autores como Berends (2011) o Kolar, Ahmad, Chan, \& Erickson (2015), quienes aconsejan el uso y la combinación de esta técnica con otras, como pueden ser las entrevistas, ya que indican que el uso exclusivo de la misma en las investigaciones sociales supone una pérdida de riqueza de los relatos individuales. Consideran que esta técnica de representación visual es benéfica y muy productiva, no obstante que matizan que habría que combinarlas con otras técnicas orales o visuales para recoger la representación en tiempo e imagen, a la misma vez que permitiría que la voz del participante sea escuchada (Berends, 2011; Kolar et al., 2015).

Ejemplos de trabajos que han empleado esta técnica de investigación podemos encontrarlos en dos vertientes: profesional e investigador. Buena muestra de los trabajos profesionales se puede localizar en trabajos de carácter profesional como los de Grissom, Czajka, \& McConnell (2015), Martin (1997), Liem y Sanders (2013) o Landgarten (1981).

En el segundo grupo, se pueden destacar los trabajos de carácter investigador de Boyd, Hill, Holmes, y Purnell (1998), Brown (2016), Frank (1984), Gramling y Carr (2004), Grissom, Czajka, \& McConnell (2015), James y Cherry (1988), Schulze (2003), Widera-Wysoczañska (1999) o Woodhouse (1992).

En el ámbito nacional y en las instituciones de Es, cabe destacar la investigación realizada por Melero, Moriña, \& López-Gavira (2018), quienes usaron las líneas de vida en el ámbito educativo para destacar las principales barreras y ayudas que los estudiantes con discapacidad identifican en las instituciones de Educación Superior. Por ello, a raíz de estos datos, así como del tratamiento y la lectura de otras investigaciones, se ha visto necesario e imprescindible el uso de esta y otras técnicas que nos conduzcan a realizar e incorporar prácticas inclusivas en el aula en esta etapa educativa (Melero, Moriña, \& López-Gavira, 2018; Moswela, \& Mukhopadhay, 2011).

\section{Diseño metodológico}

Los resultados que se presentan en este artículo pertenecen a una tesis doctoral (Suprimido revisión anónima) que se encuentra incluida dentro de un proyecto de investigación más amplio financiado por el Ministerio de Eco- 
nomía y Competitividad de España: “Suprimido para revisión anónima”. Este proyecto, con una duración de cuatro años (2011-2014), fue desarrollado por un equipo de investigación multidisciplinar compuesto por investigadores de diferentes áreas y campos de conocimiento (Ciencias de la Educación, Ciencias Económicas, Ciencias de la Salud, Ciencias Experimentales y Humanidades). El propósito principal del mismo era analizar los principales obstaculizadores y facilitadores que dos estudiantes con discapacidad identificaban en sus trayectorias universitarias en la Universidad de (suprimido para revisión anónima). De forma más específica, en la ya citada tesis doctoral (suprimido para revisión anónima) se analizó, a través de sus narraciones, la perspectiva de dos estudiantes con discapacidad en su trayectoria universitaria.

\section{Objetivo}

El propósito principal de este artículo era "Analizar los obstáculos, facilitadores y propuestas de mejora que estudiantes con discapacidad identifican en su trayectorias de formación en la universidad". Para poder profundizar en el mismo, esta tesis doctoral estableció dos objetivos esenciales:

1. Identificar, describir y explicar las barreras y ayudas que un grupo de estudiantes con discapacidad perciben en la Universidad como Institución.

2. Identificar, describir y explicar las barreras y ayudas que un grupo de estudiantes con discapacidad experimentan en las aulas universitarias.

\section{Participantes}

Esta investigación se desarrolló en una universidad del sur de España donde participaron tres estudiantes ${ }^{1}$ con discapacidad de una muestra previa de 44 con discapacidad que tomaron parte en la primera fase de la investigación anteriormente mencionada.

Para la selección de los participantes se ha seguido la selección basada en criterios de Goetz y LeCompte (1988) a través de un muestreo selectivo en el que se han tenido en cuenta los siguientes rasgos, criterios y características relevantes: estudiante con discapacidad, accesibilidad, participación, disponibilidad para participar, relevancia, voluntariedad e interés o necesidad.

${ }^{1}$ En este trabajo se van a reflejar dos relatos de los tres presentados en la investigación precedente. 
Para acceder a dicha muestra, y teniendo en cuenta la Ley de Protección de Datos II del REAL DECRETO 1720/2007, de 21 de diciembre, por el que se aprueba el Reglamento de desarrollo de la Ley Orgánica 15/1999, de 13 de diciembre, de protección de datos de carácter personal, el acceso a los mismos se realizó a través de la Unidad de Atención a Estudiantes con Discapacidad.

En el momento de la recogida de información, los dos participantes estaban estudiando diferentes titulaciones: Ingeniería y Periodismo. Respecto a su permanencia en la universidad, en el momento de la obtención de los datos, el primero de los estudiantes llevaba seis años en la universidad y el segundo cuatro.

El primero de los informantes, a quien por anonimato y protección de datos vamos a proceder a darle un nombre ficticio, ${ }^{2}$ es Andrés; en el momento de la recogida de la información tenia 27 años de edad e inició sus estudios en Ingeniería, ${ }^{3}$ correspondiente al plan del 97 en el año 2006, cambiándose, años más tarde, al actual Grado de la misma titulación. Su permanencia en la universidad es de seis años, encontrándose en segundo de grado. Su discapacidad es visual.

El segundo de los participantes, con quien se ha mantenido contacto para realizar la historia de vida en profundidad, es Rafael. ${ }^{4}$ Con 20 años de edad y una discapacidad física (distrofia muscular de Duchenne), estaba en segundo curso del Grado de Periodismo; su acceso se sitúa en el año 2011/2012 y la duración total de su carrera fue cuatro años en la universidad.

Tabla 1. Perfil de los participantes

\begin{tabular}{lccccl}
\hline Nombre & Edad & Titulación & Curso & $\begin{array}{c}\text { Años en la } \\
\text { Universidad }\end{array}$ & Discapacidad \\
\hline Andrés & 27 & G. Ingeniería & $2^{\circ}$ & 6 & Visual (Ceguera) \\
\hline Rafael & 20 & G. Periodismo & $1^{\circ} / 2^{\circ}$ & 4 & $\begin{array}{l}\text { Física (distrofia muscular de } \\
\text { Duchenne) }\end{array}$ \\
\hline
\end{tabular}

Fuente: elaboración propia

\footnotetext{
2 Siguiendo los principios éticos por los nos hemos regido en todo momento en la investigación, los nombres empleados en este trabajo han sido seleccionados por los participantes.

${ }^{3}$ Según los criterios éticos de nuestra investigación y por petición expresa de Andrés, en esta investigación no aparecerá ningún nombre real ni dato que puedan identificarlo.

${ }^{4}$ Por petición expresa, el informante ha querido mantener su nombre real.
} 


\section{Recogida de datos}

A través del uso biográfico-narrativo (Moriña, 2017b), la recogida de datos en este trabajo de investigación se ha basado en diversas técnicas cualitativas: entrevistas biográficas, entrevistas focalizadas, entrevistas semi-estructuradas, fotografías, auto-informes, línea de vida, diario de campo de la investigadora, notas de campo de la investigadora, observaciones, correos electrónicos y la técnica "Un día en la vida de...". Sin embargo, de forma concreta y específica, en este trabajo se van a presentar los datos obtenidos a través de la línea de vida, ya que se considera que esta técnica supone una revisión globalizada de los momentos más importantes en la experiencia de los participantes.

En nuestro estudio, la línea de exsistencia es presentada a los protagonistas como un eje de coordenadas donde cada uno expresa sus valoraciones en relación a los acontecimientos e hitos importante en su vida, en este caso, durante su trayectoria universitaria. Con esta técnica, se perseguía que los participantes nos indicaran un aspecto relevante o destacado vivenciado en cada curso académico. Para ello, se le ofreció al alumno un eje de coordenadas, donde en el de abcisas (X/horizontal) se establecían los cursos académicos del alumno y en el de ordenadas (Y/vertical) se medía la importancia - tanto negativa como positiva - que ese hecho tenía e incidía sobre la experiencia de los participantes. Aunque en la segunda coordenada la medición se basaba de 0 a 10, en algunos casos y momentos concretos los participantes nos propusieron si podían valorarlo de -0 a -10 .

Las líneas comienzan desde el primer año de acceso a la universidad hasta el momento actual, de forma que en la parte horizontal del eje aparecen los años y en la vertical una puntuación que se corresponderá con los hitos importantes que posteriormente serán explicados, narrados e interpretados. La línea generada corresponde con las variaciones en las que se podrán ver variaciones ascendentes o descendentes. Una vez realizada la calificación del hecho o suceso destacado y significativo para el participante, precedíamos a la unión de los puntos, finalizando en una línea donde se podía observar la trayectoria positiva o negativa del estudiante, así como los momentos importantes.

En los dos casos, aunque fue dictado y establecido por el participante, fue redactado y escrito por la investigadora por imposibilidad de ellos o, en el caso de Andrés, para facilitarle el trabajo puesto que debido a su discapacidad visual era algo que le iba a resultar complicado elaborarlo con otros medios. 
Figura 1. Línea de Vida

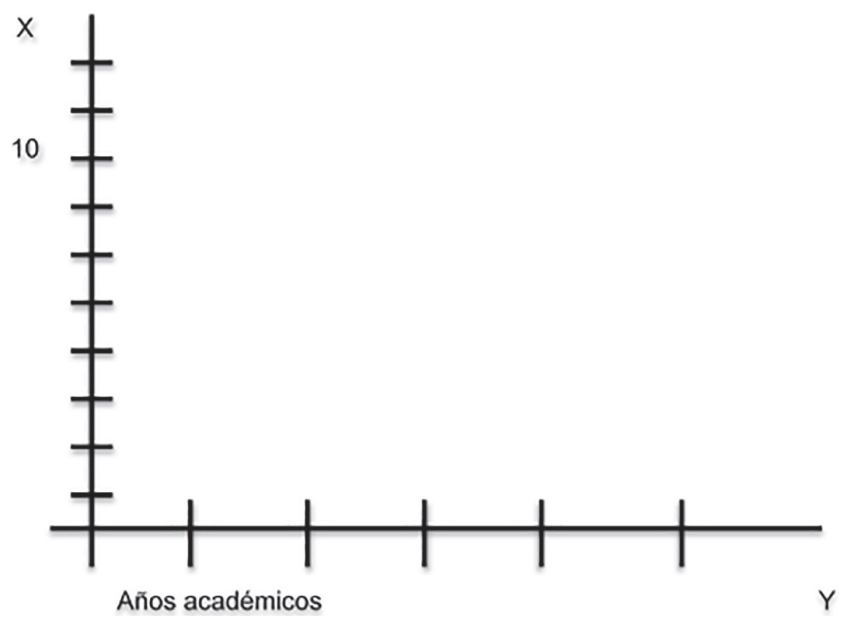

Fuente: elaboración propia

\section{Análisis de los datos}

En el proceso de la elaboración de cada narración y cada historia de vida hemos utilizado un análisis narrativo siguiendo la propuesta realizada por Goodley, Lawthom, Clough, y Moore (2004).

Persiguiendo en todo momento un punto de vista interpretativo, buscamos enfatizar la perspectiva del actor desde una postura reflexiva y comprensiva tanto sobre su experiencia como sobre los hechos, circunstancias y momentos que le llevan a tomar determinadas decisiones y/o actuaciones, por ello podemos indicar que nos basaremos en un análisis dialógico y crítico en la elaboración de las historias de vida donde el protagonista analice los acontecimientos y sucesos ocurridos en su experiencia.

La presentación de los datos de la historia se focalizará en momentos esenciales e importantes para los protagonistas, interpretando los significados de los hechos relacionándolos con el contexto social, cultural y político en el que se desarrolla.

\section{Consideraciones éticas}

Desde el punto de vista de nuestro trabajo de investigación y, en concreto, desde la investigación biográfico-narrativa, planteamos algunas considera- 
ciones éticas (Vain, 2011) que debemos tener en cuenta en la recogida de la información y, posteriormente, en su publicación. Por ello, durante todo el proceso de nuestro trabajo todos los participantes tuvieron acceso a un consentimiento informado para formar parte del estudio. Dicho consentimiento fue firmado en la primera toma de contacto en la que se les informó del procedimiento y el tratamiento con respecto a la información recopilada.

Durante todo el proceso, el estudiante ha tenido acceso a la información recogida pudiendo modificarla o suprimirla si así lo estimase oportuno. Además, se les informó que en el caso de no querer continuar adelante con el estudio iniciado, sus datos no serían tenidos en cuenta para el análisis, pasando a ser destruidos y no publicados.

\section{Resultados}

En este apartado se presentarán las historias de vida de Andrés y Rafael centradas en sus trayectorias formativas en las instituciones de Educación Superior a través de la técnica línea de vida. A lo largo del mismo, trabajaremos y analizaremos con sus narraciones personales sus vivencias y experiencias en dicha institución, analizando los procesos que ellos consideran obstaculizadores así como facilitadores.

\section{Una larga carrera de obstáculos. Historia de vida de Andrés}

Empezaré contando algo sobre mí. Me llamo Andrés, soy un chico de 27 años, nacido en un pueblo de la zona norte de Sevilla, aunque actualmente resido en la zona céntrica de esta bonita ciudad, en un piso compartido con mi hermano. Provengo de una gran familia; somos ocho hermanos, y yo soy el séptimo. Mi familia lo es todo para mí.

Nada más nacer tenía una característica que me hacía más especial si cabe al resto de los niños. Nací con un problema de visión. Sin embargo, los médicos han trabajado conmigo en numerosas operaciones para erradicar este problema, pero se ve que el objetivo no ha sido conseguido con éxito, ya que, aunque han conseguido mejorar mi visión, no se ha logrado alcanzar el objetivo al cien por cien.

Me considero una persona luchadora, constante y tenaz, que acabó cumpliendo sus objetivos sin importarme el tiempo y las batallas que tuviera que pelear para ganar, aunque en ocasiones eso llega a agotar. 
Comencé la Universidad en el año 2006, por lo que tanto había trabajado y me había esforzado. Sin embargo, no todo fueron luces en el camino, pues también existido sombras muy complicadas de pasar.

Imagen 1. Línea de vida de Andrés

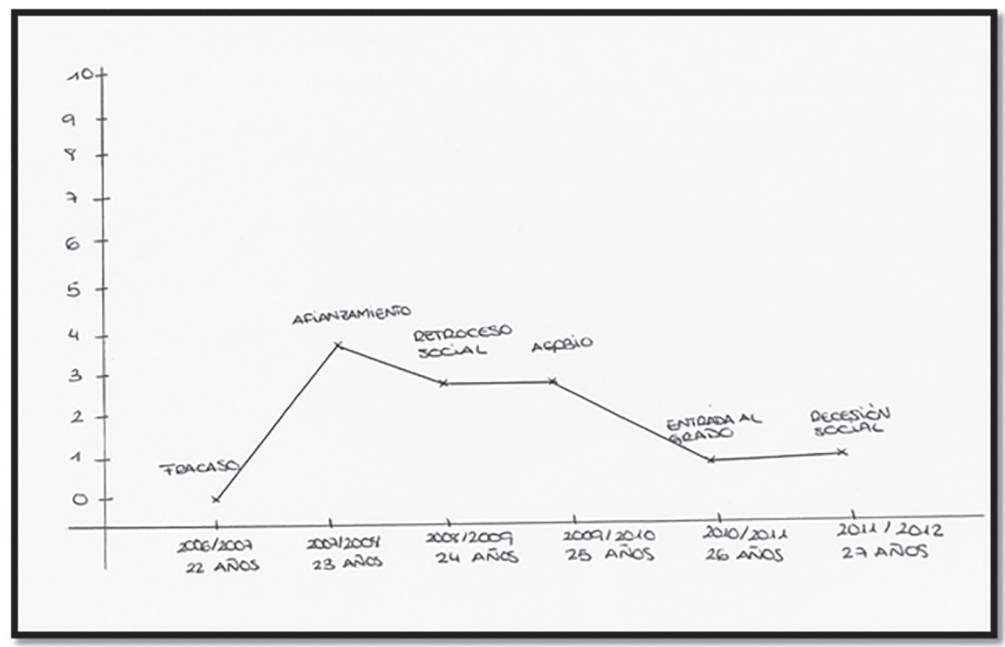

Fuente: elaboración propia

Mi trayectoria universitaria podría resumirse en la siguiente gráfica. Como puede observarse, mi trayectoria académica se caracteriza más por las barreras que me he encontrado que por las ayudas que han influido en mis estudios. Las barreras siempre se nos hacen más destacables, quizá porque se han convertido en grandes obstáculos por ser más señalados, por la dificultad de superarlo, o no sé, aunque también tengo que decir que me llevo muchas cosas positivas de mi paso por la universidad, como veremos más adelante.

Entré a la universidad con 22 años un 13 de noviembre. Yo realicé mi matricula en Informática y Gestión, pero por motivos que desconozco, la universidad me matriculó en Informática y Sistemas. Y pese a utilizar los cauces establecidos, el problema no se solventó de manera rápida y eficaz, así que perdí casi el primer cuatrimestre.

Había asignaturas en las que tenía que tener un $80 \%$ de presencialidad en las clases, y por la fecha en la que entré, eso ya era una misión imposible, por lo que ese cuatrimestre lo di por perdido. El segundo cuatrimestre lo cogí con más fuerza, pero también resultó ser desastroso: sólo aprobé una asignatura, lo que me garantizó la permanencia en los estudios.

En este cuatrimestre empezaron mis primeros problemas con la asignatura que me ha ido marcando a lo largo de mi trayectoria. El profesor se limita- 
ba a impartir las clases con transparencias manuscritas a través de la lámpara de proyección, un soporte inaccesible para mí y, pese a solicitarle los apuntes para poder estudiarlos, lo único que encontré fue una negativa por su parte.

Incluso un profesor llegó a decirme que no entendía por qué estaba allí o que no servía para estudiar esa titulación. Sin embargo, hice todo lo que pude por aprobar: fui a tutorías con dos compañeros míos, pero de nada sirvió, bueno, sí, dividió nuestro grupo y aprobó a los que a él le pareció.

Pero no todo fue negativo, pues también hice amigos que fueron una gran alianza y escudo personal para poder afrontar la situación tan negativa y complicada con la que me encontré.

En el segundo año, a diferencia del anterior, promocioné en más asignaturas y establecí una relación más íntima con los compañeros, aún así, en la asignatura problemática no conseguí pasar del 4. La situación vivida el año anterior vuelve a repetirse con otros profesores del mismo departamento, llegando incluso a solicitar tribunales para la evaluación de los exámenes. Pero estas medidas no llegaron a ningún puerto. El resto de las asignaturas evolucionaron de forma satisfactoria: los docentes se vuelcan conmigo y consigo superar hasta cuatro asignaturas.

Quién decide realizar esta carrera sabe que es similar a una factoría, la gente tiene asignaturas de diferentes cursos y, pese a repetir primero con tan sólo una asignatura de segundo, vuelvo a coincidir con muchos compañeros de primero.

Por segundo año consecutivo me adscribo al Programa de Atención de Personas con discapacidad del SACU y hago uso del servicio de cuaderno autocopiativo y del alumno colaborador ${ }^{5}$.

En mi tercer año de carrera, en el curso 2008/2009, tuve un retroceso social, me desmotivé mucho. Estaba entre dos cursos, segundo y tercero. Sólo tenía a dos compañeros de primero con los que poder relacionarme en clase, ya que no conocía a nadie y eso, unido a todos los problemas con los que mantenía con un departamento concreto, me hizo venirme abajo.

Aprobé cuatro asignaturas, pero ni qué decir tiene que con la asignatura problemática seguía suspendiéndola curso tras curso, año tras año y convocatoria tras convocatoria. Cabe destacar que, este año, recibo por parte de la ONCE una ayuda óptica electrónica, consistente en una lupa que es capaz de transmitir a un monitor o a un ordenador las imágenes que se captan a través de ella de objetos lejanos, como pizarras o pantallas de proyección. Sin em-

\footnotetext{
${ }^{5}$ Recurso que facilita el SACU al estudiante con discapacidad donde este pueda hacer uso de los servicios de otras personas para tomar apuntes, acompañamiento en los desplazamientos, acceso a la información, etc.
} 
bargo, tuve problemas para usarlas en clases, ya que alegaban que no podía grabar sin autorización y me impidieron emplearla hasta que la universidad empezó a dar a los estudiantes ayudas para la adquisición de un ordenador. A partir de ahí la situación pareció normalizarse y yo pude usar mi lupa.

En el cuarto año empiezo a agobiarme. Se anuncia la inminente llegada de los nuevos Grados. Este año yo pensaba salvar las asignaturas de los dos cursos superiores, y olvidarme un poco de la asignatura problemática de primero, pero el artículo 27 de matrícula ordenada me lo impide. Este año tengo una asignatura en $5^{\mathrm{a}}$ convocatoria, de la que no estoy matriculado y que tiene convalidación directa. Además, tengo dos asignaturas que se convalidan por una en los nuevos grados. El año siguiente, la docencia de primer curso se extingue, por lo que empiezo a olerme el grado igual que el azahar en primavera. El nuevo plan parece muy bonito, pero mi problema es que tengo la carrera ya encauzada: me faltan algunas de primero y de segundo y muy pocas de tercero.

Finalmente cambio mi matrícula al año siguiente a los nuevos grados, con todas las asignaturas problemáticas de primero ante la imposibilidad de seguir en los títulos anteriores y por la necesidad de no volver a pasar por el acceso. Ya en los nuevos grados consigo aprobar tres asignaturas de las seis en las que inicialmente me matriculé, pero ninguna de las problemáticas. La tirantez en las clases es cada día mayor.

A diferencia de años anteriores, conseguí que ese año me atendieran en tutorías quincenales, aunque en el departamento problemático esas tutorías no superaban los 10 minutos. Una anécdota de un profesor de este departamento fue cuando se encontró mi hermano en el ascensor. Le preguntó que cuántas asignaturas le quedaban para finalizar la carrera, puesto que creía tenía algunas de las suyas de primero, y cuál fue su asombro cuando mi hermano le dijo que ya había finalizado. Y lo más alucinante de todo fue la respuesta que le proporcionó “'Vaya! Ya has acabado. Tú te nos escapaste, pero el otro no se va a escapar". Eso dice mucho de todos los problemas que vengo arrastrando a mis espaldas con este departamento, pese a realizar las quejas en los órganos e instancias oportunas.

Y por fin el presente curso, 2011/2012. Consigo aprobar en el primer cuatrimestre tres asignaturas de cuatro y ipor fin! una de las problemáticas. Sin embargo, este año sigue siendo muy negativo desde el punto de vista social y tengo un retroceso, ¡mis compañeros tienen 10 años menos que yo! Y eso se nota. La conexión con ellos es más difícil, ya no existe la misma relación que con mis compañeros del primer año. 


\section{Ganas de vivir. La historia de vida de Rafa}

Imagen 2. Línea de vida de Rafa.

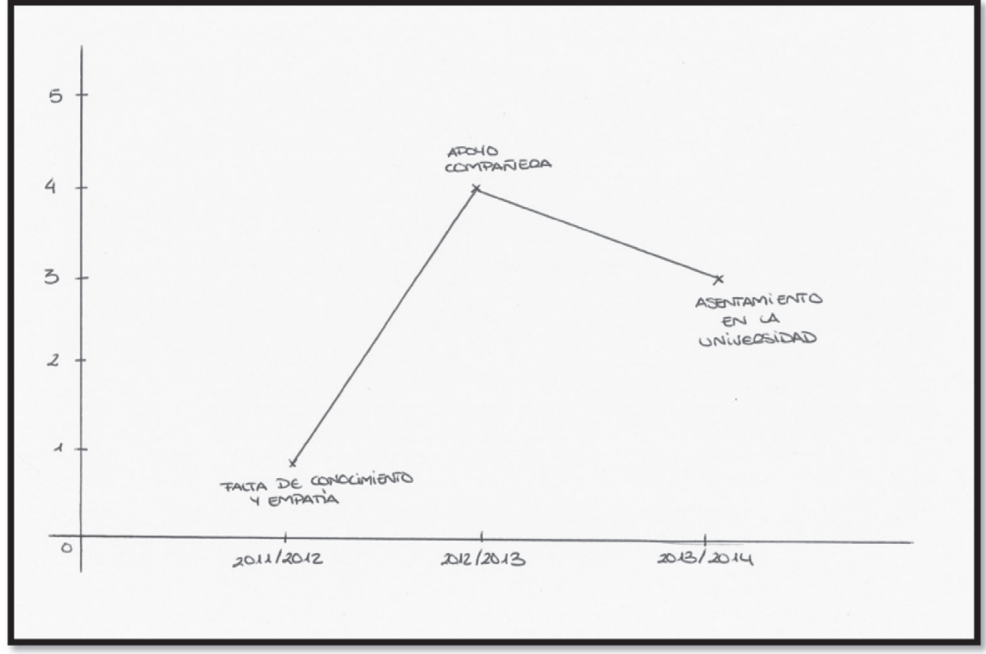

Fuente: elaboración propia

Me llamo Rafa y tengo 21 años. Vivo con mi madre y mi hermana en Dos Hermanas. Somos una familia humilde y trabajadora, todo lo que tenemos es fruto de nuestro esfuerzo y nuestro trabajo.

Me considero un chico simpático y sencillo, aunque también tímido y reservado. Sin embargo, creo que tengo que presentaros a mi colega que lleva conmigo desde los 3 años, la Distrofia Muscular de Duchenne, una enfermedad genética y degenerativa que poco a poco hace que mis músculos se vayan debilitando y desaparezcan. Mi enfermedad ha ido evolucionando a lo largo de mi vida limitando mucho mi independencia. Actualmente he perdido toda la movilidad de mi cuerpo, aunque no por ello la sensibilidad, y dependo totalmente de mi madre para cualquier actividad cotidiana: comer, moverme, vestirme, asearme... No obstante, esto no me ha hecho tener ningún tipo de complejos, a pesar de mi discapacidad. Yo siempre me he considerado una persona completamente normal al contrario, mi enfermedad me da más fuerza para superarme cada día y ser mejor persona.

Al contrario de la experiencia tan gratificante que obtuve en Selectividad, mi primer día en la Universidad fue catastrófico. Para empezar, debido a mi problema de salud entré más tarde que el resto de los compañeros, por Octubre, por lo que eso fue una barrera para mí porque la mayoría de los estudiantes ya se conocían y tenían hecho sus grupos. 
Recuerdo como si fuera ayer el primer día que entré en la Universidad, tenía pánico, miedo porque todo era completamente desconocido para mí, no sabía lo que me iba a encontrar, con quién, cómo moverme... No tuve ningún tipo de orientación en ese sentido. Lo único que sabía era el aula a donde tenía que ir, el miedo que recorría todo mi cuerpo me frenaba entrar ese día en clase. Con mis limitaciones no sabía desenvolverme y además necesitaba a mi madre para todo, al contrario que en el instituto donde siempre he estado solo, he sido independiente y ella no ha tenido que estar ya que tenía a monitores. Y eso, esa falta de autonomía, unido a lo desconocido, hizo que me bloqueara ese día.

Pero a lo largo de estos cuatro años de carrera, pese a estar con mi madre para todo y en todo, he ido superando mis barreras y mis obstáculos y puedo moverme con total libertad con la ayuda de mi madre por la Facultad. Lejos quedan ya los miedos del primer día.

Tal y como puede apreciarse en mi Línea de vida, mi primer año de universidad fue bastante negativo, vi mucho desconocimiento hacia la discapacidad por parte de los compañeros y falta de empatía. Ellos ya tenían ideas preconcebidas de lo que te pasa y te ocurre, y, para ser sinceros, no tienen ni idea. Algunos de ellos no querían ponerse conmigo en los trabajos porque pensaban que yo hacía menos que ellos... Algunos se han atrevido a decir que "i¿qué hago yo estudiando periodismo con mi discapacidad?!" Se ve que ni me conocían ni sabían de mis limitaciones. Soy capaz de quedarme un día entero sin dormir con tal de entregar mi parte, en eso soy muy responsable.

Sé que yo soy una persona tímida y que, aunque lo esté trabajando y mejorando, me suele costar relacionarme con otras personas, pero yo me esperaba otra cosa al entrar en la facultad, como por ejemplo, gente más responsable. Pero lo que vi no tiene nada que ver con lo que pensaba. Esto me hizo desmotivarme y empecé a faltar a clase porque no veía ningún tipo de compañerismo. Pero bueno, un compañero me facilitó los apuntes y en verano me puse a estudiar como un loco: aprobé cuatro de cinco.

Además, unido a la falta de compañerismo me encontré con la barrera del profesorado, el que, al verme allí, no sabía ni conocía exactamente mi situación y el motivo del por qué mi madre tenía que estar allí. Hubo profesores que incluso me lo preguntaron y no lo entendían, para ellos debía de solicitar ayuda al sAcu. Se ve que no conocían bien mi situación.

Ya conforme iban pasando los días y me iban viendo en clase, poco a poco, parece que iban comprendiendo mis necesidades, pero esto es como todo: hay profesores que te prestan toda la ayuda posible, te facilitan los recursos para poder estudiar e intentan darte unos conocimientos para que yo, después, pueda aplicarlo a mi profesión. Pero hay otros que, aún pasando años y explicándoles mis necesidades, no me facilitan nada. 
En general, puedo decir que he echado en falta asesoramiento y orientación a los profesores sobre las necesidades de las personas que posean discapacidad porque entiendo que no vas a saber de todas, pero cuando la universidad conoce que hay un alumno con determinadas necesidades en un aula, debería preparar y formar a los docentes.

Pero bueno, el tiempo pasa y llegamos al siguiente curso, el 2012/2013, año bastante positivo para mí ya que encuentro a mi gran amiga y compañera Loreto. Es a través de ella como empiezo a conocer la verdadera amistad y el compañerismo en la universidad. Me apoya y ayuda en todo, además de incluirme en todos los grupos de trabajo. Además, con ella no sólo trabajo mejor, sino que puedo participar y dar mi opinión, que eso es muy importante, y ¿sabes lo mejor de todo?, la tienen en cuenta.

A Loreto no sólo he de agradecerle la gran humanidad, su empatía y su amistad que me ha mostrado, sino también la motivación que me ha proporcionado porque, a diferencia de la gran desmotivación que tenía en el primer curso, a raíz de trabajar con ella me he implicado más en mis estudios, además de madurar y aprender muchísimo.

Aunque este año, en general, la relación con los compañeros va mejorando bastante, ya que me van conociendo y yo también a ellos. Con mi grupo de trabajo la relación es bastante buena, y con el resto digamos que me llevo bien, algunos de ellos inclusive han venido una vez a mi cumpleaños y nos juntamos en la cena de Navidad del segundo año de universidad. Sin embargo, la relación que mantenemos, pese a ser buena, es bastante superficial, nos limitamos a la relación de clase, saludarnos y poco más. El desconocimiento sigue imperante en mi aula y en toda la sociedad.

Actualmente, en este curso (2014/2015), mi leal compañera sigue a mi lado, y lo que puedo destacar como más importante, es mi asentamiento y el conocimiento de la facultad. En los cursos anteriores, puedo decir que no lo he pasado muy bien, me he encontrado desmotivado porque siempre es una batalla venir a la facultad y, eso, desgasta mucho, te cansas. Pensé que este año las relaciones con los compañeros sería diferente puesto que ya me conocían, pero no, todo sigue igual, es una relación superficial. La mayoría de las ocasiones lo achaco a mi personalidad, pero he llegado a entender que no todo es mi culpa, pienso que la gente es un poco egoísta y no se preocupa demasiado por los demás, es algo que me llama bastante la atención, porque tratándose de la facultad de comunicación, la gente debería de estar mucho más sensibilizada y concienciada de lo que ocurre a su alrededor.

Pero sí que he podido notar una evolución bastante positiva en este curso. Ya tengo más conocimiento de la mecánica y cómo funciona la universidad: la forma de estudiar, de desenvolverme, de relacionarme con los compañeros, de buscar en la biblioteca, etc. 


\section{Conclusiones y discusión}

A lo largo del presente artículo se han narrado las historias de dos estudiantes con discapacidad y su experiencia en una institución de ES a través de la técnica visual línea de vida, siendo el principal aspecto relevante éste mismo ya que, salvo trabajos mencionados (Melero, Moriña, \& López-Gavira, 2018), no abundan propuestas que empleen este instrumento en investigaciones sobre discapacidad y Educación Superior. En este sentido, y de acuerdo con Melero, Moriña, \& López-Gavira (2018), consideramos que la técnica línea de vida es una herramienta muy poderosa en la recogida de datos en la investigación cualitativa y que, por desgracia, no se hace uso de la misma en el ámbito de Ciencias Sociales. Sin embargo, desde nuestra perspectiva, consideramos que es esencial su uso y su combinación con otras técnicas que enriquezcan y complementen su significado visual dotándolo, por lo tanto, de mayor significado a través de las narraciones. Por ello, la combinación de entrevistas y/o grabaciones con la herramienta técnica línea de vida creemos que es un instrumento visual muy potente, que refleja a simple golpe de vista la trayectoria y la experiencia personal, acompañándolo, posteriormente, de significado. Conclusiones similares obtuvieron los trabajos de Berends (2011) y Kolar et al. (2015).

En concreto, y siguiendo el objetivo del presente trabajo, las narraciones de estos estudiantes han identificado en su trayectoria universitaria tanto obstáculos como facilitadores. En cuanto a las obstáculos y barreras, se puede indicar que son de dos grupos: institucionales y de aula; respecto a las ayudas, los docentes, compañeros y familiares son un referente esencial en sus trayectorias.

Referente a las institucionales, los procesos de acceso a la universidad, la incorporación del Plan de Bolonia o la falta de orientación y tutorización durante el primer año de acceso, se hace reseñable en las historias de estos estudiantes.

Respecto a esta última barrera, consideramos que sería necesario e imprescindible desarrollar planes integrales desarrollados específicamente para cada persona, en los que se implicaran y tuvieran cabida la comunidad universitaria (profesores, personal de administración y servicio y el alumnado). La creación de un profesor tutor podría ser una respuesta a la necesidad de orientación y asesoramiento con la que nuestros estudiantes se encuentran. Además, el tránsito de Bachillerato a la Universidad, y el acceso a ésta en sí, para nuestros estudiantes supuso un hito importante en su experiencia, siendo calificado este tránsito como negativo en dos de los casos. Por ello, la creación de una figura de orientación o tutor, cuyas funciones fueran guiar, 
asesorar, motivar y apoyar en el acceso y el tránsito por la Universidad, informándoles sobre las diversas cuestiones existentes, sería primordial. Al mismo tiempo, esta figura podría ser un nexo de unión entre ellos y el profesorado, al informarle sobre su discapacidad y las necesidades educativas que requieren, solventando de esta forma la inadecuada coordinación existente entre la universidad y el profesorado.

Estudios como los de Vickerman y Blundell (2010) nos hablan de esta figura de apoyo y de la importancia de la misma en los procesos de inclusión de las personas con discapacidad en la Universidad; no obstante, nos indican que únicamente ha de ser un apoyo y que el estudiante nunca ha de ser dependiente del todo.

Otro de los resultados hallados en esta investigación, es el referido al sistema de evaluación que actualmente está implantado a raíz del Plan de Bolonia, cuyas premisas se basan en un cambio de paradigma con el aprendizaje del alumno como protagonista. Sin embargo, nuestro estudio refleja que para nuestros protagonistas estas premisas no se cumplieron teniendo en ocasiones una carga de trabajo imposible de abarcar. No hay que olvidar tampoco la barrera que supone la obligatoriedad de asistencia a clase.

Dentro de este contexto, los participantes de esta investigación destacaron la figura del profesorado para garantizar prácticas educativas inclusivas. En este trabajo se remarca la necesidad imperante de una actitud positiva hacia la discapacidad por parte del profesorado, fomentando prácticas inclusivas así como la consideración de diferentes metodologías que no sean exclusivamente las lecciones magistrales. Para ello, se requiere de la realización de adaptaciones curriculares, donde se empleen varios y diferentes recursos didácticos para enseñar, usando las nuevas tecnologías de forma didáctica y formándose en temas relacionados con la discapacidad. Lo que los alumnos pedrían con esto, es que se diera una respuesta de calidad a las necesidades educativas que pudieran derivarse de sus discapacidades.

Los participantes expresaron que el apoyo recibido por parte de los docentes en ocasiones era debido a su buena voluntad, ejerciendo un impacto positivo en su motivación personal y en su rendimiento académico. Sin embargo, estas actitudes positivas eran más difíciles de conseguir, ya que dependen de la actitud y la voluntad del profesorado.

Por el contrario, en el entorno universitario estas actitudes no son siempre las más adecuadas, ya que los participantes del estudio se refirieron a cómo determinados profesores no dieron respuesta a sus problemas, sin plantear soluciones, sin realizar adaptaciones y recursos necesarios, y en algún caso en concreto las tutorías fueron excesivamente rígidas y estandarizadas. La mayoría de los estudiantes que han participado en este estudio trataron la 
respuesta ofrecida por un sector de profesores como negativas, ya que estos docentes consideraban la realización de adaptaciones curriculares suponía un trato de favor respecto a sus compañeros.

Los resultados obtenidos en este trabajo son coincidentes con investigaciones realizadas por Moswela y Mukhopadhyay (2011) o Moriña y Cotán (2017), quienes indican que, en ocasiones, algunos profesores se niegan a realizar adaptaciones curriculares, ya que consideran que se baja el nivel de enseñanza o supone un trato de favor frente a otros compañeros sin discapacidad. Tal y como indican Olney y Brockelman (2003), el desconocimiento de la discapacidad puede llegar en ocasiones a modificar las percepciones y no entender las adaptaciones como un trato de favor sino como una necesidad y una obligatoriedad según la legislación.

No obstante, debemos especificar que los dos estudiantes han tenido diferentes experiencias en materia de evaluación, ya que, en la mayoría de los casos, los profesores han accedido a modificar fechas, duración y tipo de exámenes para satisfacer sus necesidades. En las ocasiones que se han tomado estas actuaciones, han sido valoradas de forma positiva por parte de los estudiantes. Resultados similares se han encontrado en trabajos realizados por Tinsley y Beale (2010), quienes indicaron que el asesoramiento, la orientación o la adaptación curricular - como el tiempo extra en los exámenes - , son actitudes positivas que los estudiantes encuentran como una gran ayuda para la continuidad de sus estudios, incidiendo de forma satisfactoria en sus expectativas académicas.

Otra de las cuestiones que nos gustaría resaltar, es el sobresfuerzo que estos estudiantes tuvieron que realizar para poder superar sus retos académicos. Para la mayoría de ellos, la universidad les aportó nuevas oportunidades en la vida y nuevos caminos laborales, sin embargo, este camino, en ocasiones, no es fácil. Para ellos, sus trayectorias educativas y vitales se encontraron marcadas por obstáculos y situaciones que debían ir solventando $\mathrm{y}$, pese a que consiguieron los objetivos establecidos, el trabajo que tuvieron que realizar para ello fue duro y largo en el tiempo.

Otro de los datos revelados en este trabajo, hace alusión a las estrategias personales y redes familiares, sociales y académicas que les han permitido hacer frente a las adversidades (Duggan \& Linehan, 2013; Cotán, 2017). Trabajos como los de Ewert y Yoshino (2011), Sandín et al. (2014) o Silva y Jiménez (2015), indican que el apoyo social, la confianza, la responsabilidad, el sentido de logro, la perseverancia y la influencia del contexto, entre otros, son los principales factores de resiliencia que ayudan a los estudiantes a afrontar los numerosos obstáculos y barreras con los que se encuentran en el contexto universitario y mejoran la calidad de vida del estudiante. 
Desde este trabajo, se ha analizado y hecho mención a las redes académicas (personal de administración y servicio, profesorado, compañeros y Unidad de Atención al Estudiante con Discapacidad), así como a las ayudas que el SACU ha realizado. En estudios previos como los de Cotán (2017), Gibson (2012) o Silva y Jiménez (2015), se hace mención de algunos de estos apoyos como elementos facilitadores para la inclusión de los estudiantes con discapacidad. En el caso concreto de Andrés, se ha referido a una red de apoyo organizado como es la ONCE. Destaca la ayuda recibida en cuanto a la facilitación y la transcripción en braille de los materiales empleados en clase.

La relación que los estudiantes mantuvieron con sus compañeros de aula y el papel que han jugado otras personas (familiares, amigos, etc.) durante sus trayectorias universitarias marcaron de forma decisiva sus experiencias académicas, aunque en algunos casos estas experiencias hayan sido negativas. Respecto a los compañeros, las narraciones lo explicaron tanto en términos de barrera como de ayuda. Como barrera se identificaron aquellos compañeros con los que no mantenían ningún tipo de relación, además de los prejuicios generados por la falta de información sobre sus necesidades. Respecto a los compañeros que fueron identificados como ayuda, señalaron que son aquellos que eran empáticos ante sus necesidades, que mostraron una actitud abierta y flexible y les ayudaron con su aprendizaje (apuntes, trabajos en grupos, etc.). Este tipo de compañeros se convirtieron en un pilar fundamental en su trayectoria, resultando como factores protectores que le facilitaron hacer frente a las adversidades.

En este sentido, para que la Es fuera resiliente debería de centrarse tanto en curricula y resultados académicos, así como en crear iniciativas para propiciar contextos universitarios que generen espacios y tiempo que puedan dedicarse a profundizar y desarrollar relaciones humanizadas y no meramente académicas. En definitiva, siguiendo a Fuller, Bradley, y Healey (2004) o Silva y Jiménez (2015), pensamos que debería hacerse mayor énfasis en los aspectos sociales y emocionales del aprendizaje, potenciando aquellos factores que generan y favorecen la inclusión social y educativa.

Por ello, se puede afirmar que las instituciones de ES, en el caso concreto de las personas con discapacidad, supone una oportunidad y opciones de mejora y empoderamiento de sus propias vidas (Fuller et al., 2004; Guzmán, 2017; Moswela \& Mukhopadhyay, 2011). No obstante, tan sólo el 8\% de este colectivo tiene estudios universitarios. Un tránsito y una experiencia que en ocasiones se ven teñidos por los obstáculos y barreras con los que tienen que enfrentarse para aprender y participar en la vida institucional universitaria (Fuller et al.,2004; Gibson, 2012; Vickerman \& Blundell, 2010). 


\section{Referencias Bibliográficas}

Baglieri, S., Valle, J., Connor, D. J., \& Gallagher, D. (2011). Disability studies and special education: The need for plurality of perspectives on disability. Remedial and Special Education, 32(4), 267-278. doi: 10.1177/0741932510362200

Berends, L. (2011). Embracing the visual: using timelines with in-depth interviews on substance use and treatment. The Cualitative Report, 16(1), 1-9. Disponible en https://nsuworks.nova.edu/tqr/vol16/iss1/1

Boyd, C. J., Hill, E., Holmes, C., \& Pur- nell, R. (1998). Putting drug use in context: Lifelines of African American women who smoke crack. Journal of Substance Abuse Treatment, 15(3), 235-249. doi: 10.1016/S0740-5472(97)00195-5

Brott, P. E. (2001). The storied approach: A postmodern approach to career counseling. The Career Development Quarterly, 49, 304-313. doi: 10.1002/j.21610045.2001.tb00958.x

Brown, A. (2016). An exploratory study investigating the impact of a university module that aims to challenge students' perspectives on ageing and older adults. Practitioner Research in Higher Education Journal, 10(2), pp.25-39. Disponible en https:/ / files.eric.ed.gov/fulltext/EJ1129960.pdf

Cotán, A. (2015). Enseñanza Superior y Educación Inclusiva: múltiples miradas desde las historias de vida de estudiantes con discapacidad (Tesis doctoral) Universidad de Sevilla, Sevilla. Disponible en https://idus.us.es/xmlui/handle/11441/32051

Cotán, A. (2017). Educación inclusiva en las instituciones de educación superior: narrativas de estudiantes con discapacidad. Revista Española de Discapacidad, 5(I): 43-61. doi: 10.5569/2340-5104.05.01.03

Cruz, R. \& Casillas, M.Á. (217). Las instituciones de Educación Superior y los estudiantes con discapacidad en México. Revista de la Educación Superior, 46(181), 37-53. Doi: 10.1016/j.resu.2016.11.002

Duggan, C., \& Linehan, C. (2013). The role of natural supports in promoting independent living for people with disabilities: A review of existing literature. British Journal of Learning Disabilities, 41, 199-207. doi: 10.1111/bld.12040

Ewert, A., \& Yoshino, A. (2011). The influence of short-term adventure-based experiences on levels of resilience. Journal of Adventure Education and Outdoor Learning, 11(1), 35-50. doi: 10.1080/147229679.2010.532986

Fossey, E., Chaffey, L., Venville, A., Ennals, P., Douglas, J., \& Bigby, C. (2017). Navigating the complexity of disability support in tertiary education: perspectives of students and disability service staff. International Journal of Inclusive Education, 21(8), 822-832. doi: 10.1080/13603116.2017.1278798

Frank, G. (1984). Life history model of adaptation to disability: The case of a "congenital amputee." Social Science and Medicine, 19(6), 639-645. doi: 10.1016/0277-9536(84)90231-4

Fuller, M., Bradley, A., \& Healey, M. (2004). Incorporating disabled students within an inclusive higher education environment, Disability \& Society, 19(5), 455-468. doi: 10.1080/0968759042000235307

Fundación Universia (2012). Guía de Atención a la Discapacidad en la Universidad 2012 (en línea). Recuperado de http://www.fundacionuniversia.net/wp-content/ uploads/2016/03/GUIA-ATENCION-A-LA-DISCAPACIDAD-WEB-Def.pdf 
Fundación Universia (2013). Guía de Atención a la Discapacidad en la Universidad 2013 (en línea). Recuperado de http://www.fundacionuniversia.net/wp-content/ uploads/2016/03/GUIA_ATENCION_A_LA_DISCAPACIDAD_ACC2013.pdf

Fundación Universia (2014). ). Guía de Atención a la Discapacidad en la Universidad 2014 (en línea). Recuperado de http:/ / www.fundacionuniversia.net/wp-content/uploads/2016/03/GUIA-ATENCION-A-LA-DISCAPACIDAD_14_P.pdf

Fundación Universia (2015). Guía de Atención a la Discapacidad en la Universidad 2015 (en línea). Recuperado de http://www.fundacionuniversia.net/guiade-atencion-a-la-discapacidad-en-la-universidad-2015/

Fundación Universia (2016). Guía de Atención a la Discapacidad en la Universidad 2016 (en línea). Recuperado de http://www.fundacionuniversia.net/wp-content/ uploads/2016/03/Guia_Atencion_Discapacidad_2016_ACCESIBLE.pdf

Fundación Universia (2017). Guía de Atención a la Discapacidad 2017 (en línea). Recuperado de http://www.fundacionuniversia.net/wp-content/uploads/2017/05/ Guia_Atencion_Discapacidad_2017_ACCESIBLE.pdf

Gibson, S. (2012). Narrative accounts of university education: socio-cultural perspectives of students with disabilities. Disability \& Society, 27(3), 353-369. doi: 10.1080/13603116.2015.1015177

Goetz J.P., \& LeCompte, M.D. (1988). Etnografía y diseño cualitativo en investigación cualitativa. Madrid: Morata.

Goodley, D., Lawthom, R., Clough, P., \& Moore, M. (2004). Researching life stories. London: Routledge.

Gramling, L. F., \& Carr, R. L. (2004). Lifelines. A Life History Methodology. Nursing Research, 53(3), 207-210. doi: 10.1097/00006199-200405000-00008

Grissom, A. N., Czajka, C. D. \& McConnell, D. A. (2015). Revisions of physical geology laboratory courses to increase the level of inquiry: Implications for teaching and learning. Journal or Geosciencie Education, 63(4), 285-296. doi: 10.5408/14-050.1

Guzmán, C. (2017). Las nuevas figuras estudiantiles y los múltiples sentidos de los estudios universitarios. Revista de la Educación Superior, 46(182), 71-87. Doi: http:/ / dx.doi.org/10.1016/j.resu.2017.03.002

Hurst, A. (2015). Developing and implementing an accreditation scheme for disability services staff in post-compulsory education in the United Kingdom. Journal of Postsecondary Education and Disability, 28(1), 9-24. Disponible en https:/ / files.eric.ed.gov/fulltext/EJ1066321.pdf

Jacklin, A., Robinson, C., O'Meara, L., \& Harris, A. (2007). Improving the experiences of disabled students in higher education. Disponible de http:/ / www. heacademy.ac.uk/assets/documents/research/jacklin.pdf

James, J., \& Cherry, E. (1988). The grief recovery handbook: A step-by-step program for moving beyond loss. New York: Harper \& Row

Kilpatrick, S., Johns, S., Barnes, R., Fischer, S., McLennan, D., \& Magnussen, K. (2017). Exploring the retention and success of students with disability in Australian higher education. International Journal of Inclusive Education, 21(7), 747762. doi: 10.1080/13603116.2016.1251980

Kolar, K., Ahmad, F., Chan, L., \& Erickson, P.G. (2015). Timeline mapping in qualitative interviews: A study of resilience with marginalized groups. International Journal of Qualitative Methods, 14(3), 13-32. doi: 10.1177/160940691501400302 
Landgarten, H.B. (1981). Clinical art therapy. New York: Bninner-Mazel.

Liem, A. \& Sanders, E. (2013). Human-centred Design Workshops in Collaborative Strtegizc Design Projects: An educational and profesional comparison. Design and Technology Education: An Internacional Journal, 18(1), 72-86. ISSN 13601431. Disponible en https://ojs.lboro.ac.uk/DATE/article/view/1801/1735

Martin, E. M. (1997). The Symbolic Graphic Life-Line: Integrating the past and Present through Graphic Imagery, Art Therapy. Journal of the American Art Therapy Association, 14(4), 261-267. doi: 10.1080/07421656.1987.10759295

Melero, N., Moriña, A., López-Gavira, R. (2018). Life-lines of students with disabilities during their university trajectories. The Qualitative Report, 23(5), p. 1127-1145. Disponible en https:/ / nsuworks.nova.edu/tqr/vol23/iss5/7/

Morgado, B., López, R., \& Moriña, A. (2017). The ideal university classroom: Stories by students with disabilities. International Journal of Educational Research, 85, 148-155. doi: 10.1016/j.ijer.2017.07.013

Moriña, A. (2017a). Inclusive education in higher education: challanges and opportunities. European Journal of Special Needs Education, 32(1), 3-17. doi: 10.1080/08856257.2016.1254964

Moriña, A. (2017b). Investigar con Historias de Vida. Metodología biográfico-narrativa. Madrid: Narcea.

Moriña, A. \& Cotán Fernández, A. (2017). Educación Inclusiva y Enseñanza Superior desde la Mirada de Estudiantes con Diversidad Funcional. Revista Digital de Investigación en Docencia Universitaria, 11(1), 19-35. doi: 10.19083/ ridu.11.528

Moswela, E., \& Mukhopadhyay, S. (2011) Asking for too much? The voices of students with disabilities in Botswana. Disability $\mathcal{E}$ and Society, 26(3), 307-319. doi: 10.1080/09687599.2011.560414

Okocha, A. G. (1998). Using qualitative appraisal strategies in career counseling. Journal of Employment Counseling, 35, 151-159. doi: 10.1002/j.2161-1920.1998. tb00996.x Olney, M.J., \& Brockelman, K.F. (2003). Out of the Disability Closet: Strategic use of perception management by select university students with disabilities. Disability $\mathcal{E}$ Society, 18(1), 35-50. doi: 10.1080/713662200

Pliner, S., y Johnson, J. (2004). Historical, theoretical, and foundational principles of universal instructional design in higher education. Equity $\mathcal{E}$ Excellence in Education, 3, 105-113. doi: 10.1080/10665680490453913

Rodríguez, A., \& Álvarez, E., (2014). Estudiantes con discapacidad en la Universidad. Un estudio sobre su inclusión. Revista Complutense de Educación, 25(2), 457-479. doi: 10.5209/rev_RCED.2014.v25.n2.41683

Ruiz Hidalgo, C. (2015). Tutorización de un alumno con síndrome de asperger: coordinación y evaluación de los estudios de Grado en Derecho. Revista de Educación y Derecho, 11, 1-17. Disponible en http://revistes.ub.edu/index. $\mathrm{php} /$ RED/article/view/11997/14775

Sandín, Ma P., Bazoco, J., Del Campo, J., Forés, A., Massot, I., Sacramento, R., \& Sánchez, A. (2014). Resiliència, immigraciò i èxit escolar. Temps d'Educació, 46, 11-31. Disponible en https://ddd.uab.cat/pub/artpub/2014/169864/temedu_a2014n46p11.pdf

Schulze, A. (2003). Graphic Life Map. Yankton: MarcoPolo Education Fundation. Disponible en https://eric.ed.gov/?id=ED481424 
Shaw, J. A. (2009). Chance happenings in life and psychotherapy. Psychiatry, 72(1), 1-12. doi: 10.1521/psyc.2009.72.1.1.

Silva, Y.M., \& Jiménez, A. (2015). Estudiantes de contextos vulnerables en una universidad de élite. Revista de Educación Superior, 44(175), 95-119. ISSN 2395-9037. Disponible en http://resu.anuies.mx/ojs/index.php/resu/ article/view/158/129

Strnadová, I., Hájková, V., \& Kve $\square$ ton $\square$ ová, L. (2015). Voices of university students with disabilities: inclusive education on the tertiary level - a reality or a distant dream? International Journal of Inclusive Education, 19(10), 1080-1095. doi: $10.1080 / 13603116.2015 .1037868$

Thomas, L. (2016). Developing inclusive learning to improve the engagement, belonging, retention, and success of students from diverse groups. En M. Shah, A. Bennett, \& E. Southgate (Eds.), Widening higher education participation. A global perspective (pp. 135-159). Oxford, UK: Elsevier.

Tinsley, B., \& Beale, M. (2010). High hope and low regard: The resilience of adolescents' Educational Expectations while developing in challenging political contexts. Research in Human Development, 7(3), 183-201. doi: http:/ / dx.doi.org /10.1080/15427609.2010.505780

Vain, P.D. (2011). La , ICE, Revista Electia como naturalizacicia y Cambio en Educacios que se puedan obtener y que puedan dañar a los dempectos ciética en la investigación educativa y el riesgo del uso de la ciencia como naturalización de lo social. REICE, Revista Electrónica Iberoamericana sobre Calidad, Eficacia y Cambio en Educación, 9(2), 15-23. Disponible en http://www.redalyc.org/articulo.oa?id=55119127002

Vander Kloet, M. (2015). Accessibility in Teaching Assistant Training: A Critical Review of Programming from Ontario's Teaching and Learning Centres. The Canadian Journal for the Scholarship of Teaching and Learning, 6(2). doi: . 10.5206/ cjsotlrcacea.2015.2.9

Vickerman, P., \& Blundell, M. (2010). Hearing the voices of disabled students in higher education. Disability $\mathcal{E}$ Society, 25(2), 21-32. doi: $10.1080 / 09687590903363290$

Widera-Wysoczañska, A. (1999). Everyday Awareness of Death: A Qualitative Investigation. Journal of Humanistic Psychology, 39, 73-95. doi: $10.1177 / 0022167899393008$

Woodhouse, L. D. (1992). Women with jagged edges: Voices from a culture of substance abuse. Qualitative Health Research, 2(3), 262-281. doi: $10.1177 / 104973239200200302$

Yssel, N., Pak, N. \& Beilke, J. (2016). A Door Must Be Opened: Perceptions of Students with Disabilities in Higher Education. International Journal of Disability, Development and Education, 63(3), 384-394. doi: 10.1080/1034912X.2015.1123232 
